\title{
Treatment Results of Adjuvant Brachytherapy as Monotherapy in Endometrial Cancer: A Retrospective Study from Faculty of Medicine, Chiang Mai University
}

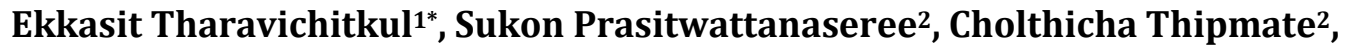 \\ Somvilai Chakrabandhu1, Pitchayaponne Klunklin1, Wimrak Onchan1, \\ Razvan M. Galalae ${ }^{3}$, Imjai Chitapanarux ${ }^{1}$ \\ ${ }^{1}$ The Division of Therapeutic Radiology and Oncology, Faculty of Medicine, Chiang Mai University, Chiang Mai, \\ Thailand \\ ${ }^{2}$ Biostatistics and Applied Statistics Laboratory, Department of Statistics, Faculty of Science, Chiang Mai \\ University, Chiang Mai, Thailand \\ ${ }^{3}$ Faculty of Medicine, Christian-Albretch University, Kiel, Germany \\ Email: paan 31@hotmail.com
}

Received 7 December 2014; revised 25 December 2014; accepted 31 December 2014

Academic Editor: Sibu P. Saha, University of Kentucky, USA

Copyright (C) 2015 by authors and Scientific Research Publishing Inc.

This work is licensed under the Creative Commons Attribution International License (CC BY).

http://creativecommons.org/licenses/by/4.0/

(c) ()

\begin{abstract}
Purpose: To report the retrospective study of using intravaginal brachytherapy as adjuvant monotherapy for endometrial cancer. Materials and Methods: From 2001-2009, 47 patients who received completely surgical staging for endometrial carcinoma and were designed by multidisciplinary team were enrolled. All patients received intravaginal brachytherapy (IVBT) with the dose of 5.5 - $7 \mathrm{~Gy}$ in 2 - 6 fractions. The treatment results and late toxicities were evaluated and recorded. Results: At the median follow-up time of 44 months, the local control, disease-free survival, metastasis-free survival and overall survival rates were $100 \%, 97.9 \%, 97.9 \%$ and, $97.9 \%$, respectively. Only age showed the statistical significance with the p-value of 0.046 . Two patients $(4.3 \%)$ developed late genitourinary toxicity. Conclusion: The using of adjuvant IVBT as monotherapy for endometrial carcinoma is feasible.
\end{abstract}

\section{Keywords}

Endometrial Cancer, Adjuvant, Brachytherapy, Monotherapy

\footnotetext{
${ }^{*}$ Corresponding author.
}

How to cite this paper: Tharavichitkul, E., et al. (2015) Treatment Results of Adjuvant Brachytherapy as Monotherapy in Endometrial Cancer: A Retrospective Study from Faculty of Medicine, Chiang Mai University. Journal of Cancer Therapy, 6, 34-39. http://dx.doi.org/10.4236/jct.2015.61005 


\section{Introduction}

Endometrial Cancer was one of the gynecological cancers in Thailand. From the study of Tangjitgamol et al., the most common symptom was abnormal uterine bleeding (87.3\%) and 78\% of them had early stage disease [1].

Surgery (Total abdominal hysterectomy \& Bilateral salpingo-oophorectomy: TAH \& BSO) \pm pelvic lymph node dissection (PLND)) is the primary treatment for endometrial cancer. For further treatment, four randomized studies demonstrated a reduction of pelvic recurrences with addition adjuvant external beam radiotherapy following surgical treatment. The most benefit of radiotherapy is to reduce recurrence at the vaginal cuff, the most common site of failure [2]-[5].

The alternative radiotherapy to reduce the toxicity from irradiation is intravaginal brachytherapy (IVBT). According to the publication of the second post-operative radiation therapy in endometrial cancer (PORTEC-2) study, the results of this study reported that IVBT was no difference of vaginal cuff recurrence in comparison to whole pelvic radiotherapy (WPRT) in high-intermediate risk group as defined by Gynecologic Oncology Group study No. 99 (GOG 99). Moreover, PORTEC 2 study reported the less toxicity profiles in the patients who received IVBT as sole treatment [6].

In our institution, IVBT has been used to treat early stage endometrial cancer. This retrospective study was designed to evaluate the treatment results of adjuvant IVBT alone for endometrial carcinoma in Faculty of medicine, Chiang Mai University.

\section{Materials and Methods}

After the acceptance of ethical board review, patients who had $\geq 18$-year-old, pathologically proven endometrioid adenocarcinoma with intermediate to high risk for recurrences, stage I-III according to the International Federation of Gynecology and Obstetrics (FIGO) staging edition of 1998. Complete surgical staging (Total abdominal hysterectomy \& Bilateral salpingo-oophorectomy: TAH\&BSO) plus pelvic lymph node dissection (PLND)) was performed and the pathological results were reviewed and designed to receive adjuvant IVBT as monotherapy according to the consensus of multidisciplinary team composed of gynecologic oncologist and radiation oncologist. From the year of 2001 to 2009, there were 199 patients of endometrial cancer who were treated and 47 patients were designed to treat by IVBT alone.

According to anatomy of vaginal cuff, either intra-vaginal cylinder or vaginal ovoids were applied to the areas of vaginal stump and upper third of vaginal mucosa with the prescribed dose at $5 \mathrm{~mm}$ from applicator surface. All patients received IVBT with the dose of 5.5 - 7 Gy in 2 - 6 fractions.

After the treatment finished, patients were scheduled to visit for per vaginal examination (PV exam) in the follow-up program. The follow-up program schedule was every 3 months in the first 3 years after treatment, 6 monthly in years 4 and 5, then annually after five years. A vaginal examination was performed to evaluate the disease status according to World Health Organization (WHO) criteria [7]. Investigations (tissue biopsy, medical imaging or laboratories) for disease progression were performed as indicated when patients presented with suggested symptoms. Late toxicities were evaluated according to the Radiation Therapy Oncology Group/European Organization of Research and Treatment of Cancer (RTOG/EORTC) late toxicity criteria [8].

All descriptive and qualitative analyses were evaluated. Survival analysis data were calculated by KaplanMeier method and log-rank test and univariate analysis were used to measure the relationship between patient factors to the treatment results using Cox's proportional hazard model [9]-[11].

\section{Results}

From 2001-2009, 47 patients were enrolled to this study. The mean age of patients was 59 years old. The pathologic results reported of all patients were endometrioid adenocarcinoma. Eighteen patients (38\%) were stage IC. Forty-four percents were well-differentiated grade. The mean total treatment time was 14 days. Seventeen patients (36.2\%) received the dose schedule of $4 \times 6$ Gy. Characteristic data were showed in Table 1 .

At the median follow-up time of 44 months (IQR; 12 - 72 months), the local control, disease-free survival, metastasis-free survival and overall survival rates were 100\%, 97.9\%, 97.9\% and, 97.9\%, respectively. All survival data were shown in Figure 1 and Figure 2.

From univariate analysis, stage, myometrial invasion, grade, cervical involvement and age were evaluated. Only age showed the statistical significance with the p-value of 0.046 . No statistical significance could be observed on other parameters (Table 2). 


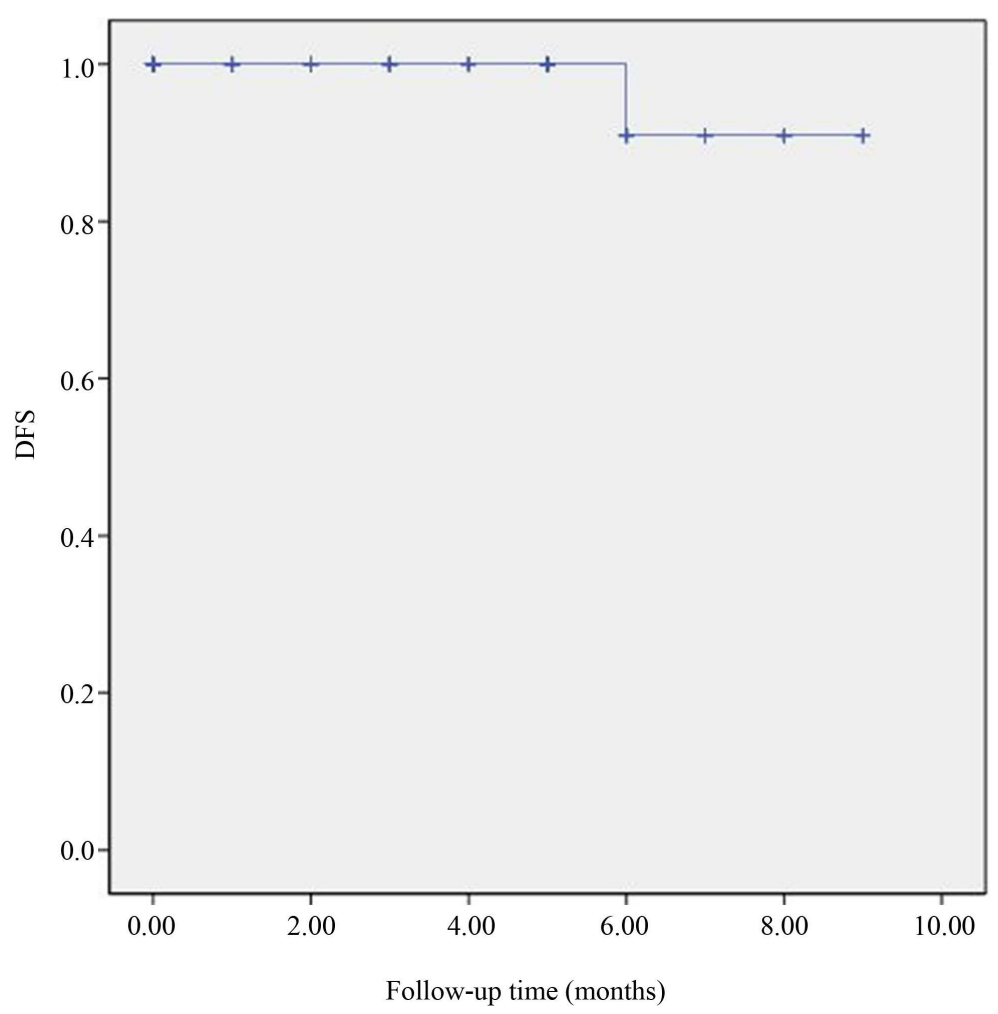

Figure 1. Kaplan-Meier curve showed disease-free survival rate.

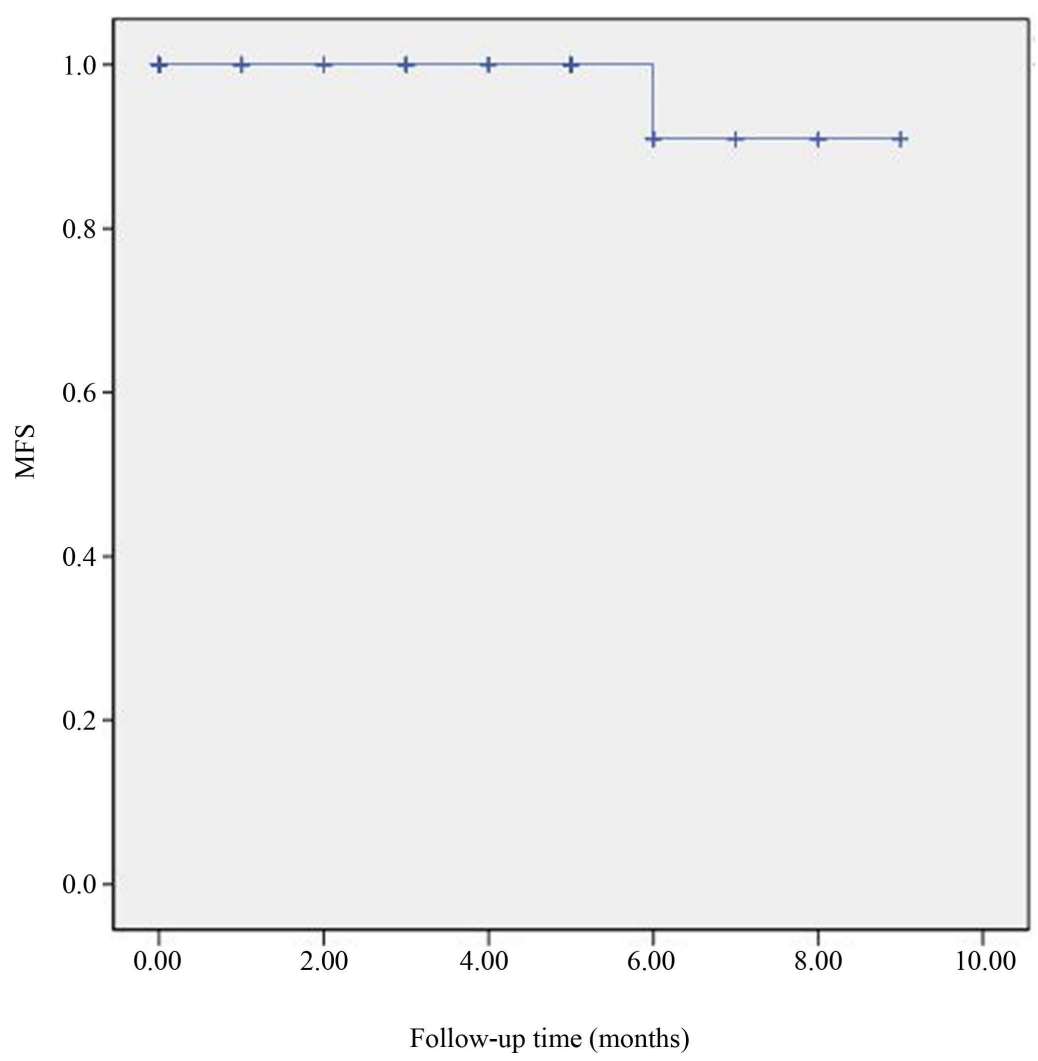

Figure 2. Kaplan-Meier curve showed metastasis-free survival rate. 
Table 1. Characteristics data of these patients.

\begin{tabular}{cc}
\hline Parameters & Numbers (total N = 47) \\
\hline Age (years) & Mean (SD) 59.09 (9.60) \\
Median (IQR) $60.00(12.00)$ \\
Stage \\
Ib \\
Ic \\
IIA & $12(25.5)$ \\
IIB & $18(38.3)$ \\
IIIA & $12(25.5)$ \\
Grade & $4(8.5)$ \\
Well-differentiated (grade 1) & $1(2.1)$ \\
Moderately differentiated (grade 2) & \\
Poorly differentiated (grade 3) & $21(44.7)$ \\
Others & $16(34.0)$ \\
Myometrial invasion & $9(19.1)$ \\
Yes & $1(2.1)$ \\
No & \\
Cervical involvement & $25(53.2)$ \\
Yes & $22(46.8)$ \\
No & \\
Total treatment time (days) & $26(55.3)$ \\
Dose schedules & $21(44.7)$ \\
$4 \times 6$ Gy & \\
$3 \times 7$ Gy & $\times 6$ Gy \\
Others & Mean (SD) 14.34 (7.46) \\
\hline
\end{tabular}

Notes: $\mathrm{SD}=$ standard deviation, IQR = inter-quartile range.

Table 2. Uni-variate analysis for IVBT as monotherapy.

\begin{tabular}{|c|c|c|c|}
\hline Variables & HR & $95 \% \mathrm{CI}$ & $\mathrm{p}$-value \\
\hline \multicolumn{4}{|l|}{ Stage } \\
\hline Ia, b, c & 1.000 & - & \\
\hline \multirow[t]{2}{*}{ IIa, b \& IIIa } & 0.499 & $0.104-2.397$ & 0.386 \\
\hline & Myometria & & \\
\hline Yes & 0.820 & $0.205-3.275$ & 0.778 \\
\hline No & 1.000 & - & \\
\hline Grade & & & 0.982 \\
\hline Well-differentiated & 0.850 & $0.088-8.251$ & 0.889 \\
\hline Moderately differentiated & 0.798 & $0.078-8.212$ & 0.849 \\
\hline Poorly differentiated & 1.000 & - & \\
\hline \multicolumn{2}{|c|}{ Cervix involvement } & & 0.186 \\
\hline Endocervical gland & 1.168 & $0.311-4.381$ & 0.818 \\
\hline Stromal & 1.644 & $0.179-15.070$ & 0.660 \\
\hline None & 1.000 & - & \\
\hline \multicolumn{4}{|l|}{ Age } \\
\hline Less than 60 & 1.000 & - & \\
\hline More than 60 & 8.218 & $1.034-65.313$ & 0.046 \\
\hline
\end{tabular}


For the toxicity profiles, two patients (4.3\%) developed late Genitourinary toxicity and no patients developed late Gastrointestinal toxicity (Table 3).

\section{Discussions}

Adjuvant radiotherapy for endometrial cancer is typically offered to patients with adverse factors in order to decrease the risk of relapse and most of supported data for adjuvant radiotherapy came from WPRT [2]-[5]. Because vaginal cuff is the most common site of recurrence, IVBT that treat directly to this area may be an option with less toxicity. Hong et al. reported sixty-nine women of FIGO stages I and II endometrial cancer underwent adjuvant irradiation with IVBT. At 45 months of median follow-up time, the 5-year disease-free survival was 92\% and the overall survival rate was $79 \%$. Multivariate Cox regression analysis reported that grade, age, and stage were significant predictors of survival. The overall acute and late side effects were minimal [12]. Chadha et al. reported 124 patients with stage IBG3 and IC endometrial carcinoma were treated postoperatively with highdose-rate vaginal vault brachytherapy as the only adjuvant treatment during February 1991 to August 1997. A dose of $3 \times 7$ Gy was delivered to $5 \mathrm{~mm}$ from the applicator. At median follow-up time of 30-month, no patient has developed a vaginal or pelvic recurrence. Three patients developed tumor recurrence. The 5-year actuarial overall survival and disease-free survival are $93 \%$ and $87 \%$, respectively. There was no treatment-related grade 3 or 4 morbidity observed [13]. McCloskey et al. reported the using of vaginal cuff brachytherapy alone in 77 patients with high-intermediate risk group. The vaginal failure rate was $1.2 \%$ and pelvic failure rate was $3.4 \%$ [14]. Eldredge-Hindy et al. reported using adjuvant high-dose rate vaginal brachytherapy for eighty-six patients with high-intermediate risk (HIR) and high-risk, early stage endometrial cancer. They reported four recurrences, including one vaginal recurrence. The 3-yr vaginal, pelvic and distant recurrences were $1.9 \%, 2.4 \%$ and $9.1 \%$, respectively. The 3-yr disease-free and overall survival rates were $87.7 \%$ and $93.9 \%$, respectively [15].

Because the phase 2 data suggesting IVBT was as effective as external beam radiotherapy in reducing the incidence of vaginal recurrence, the phase 3, PORTEC-2, trial was devised to answer the question of whether IVBT is sufficient treatment to prevent vaginal recurrence. Patients who had the high intermediate-risk group with the following features were included in the study; age greater than 60 and stage IC grade 1 or 2, or stage IB grade 3, stage IIA (except grade 3 extending into the outer half of the myometrium). Over 400 patients were randomized to either external beam radiotherapy or vaginal cuff brachytherapy. There was no significant difference in rates of vaginal and pelvic recurrence at three years which were $2 \%$ and $3.5 \%$ in the brachytherapy arm versus $1 \%$ and $0.6 \%$ in the external beam arm. Distant relapse rates were $5.7 \%$ with external beam versus $6.3 \%$ with brachytherapy. Toxicity and quality of life were evaluated and showed better outcome with IVBT as monotherapy [6].

From our study, the 4-year local control, disease-free, metastasis-free survival and overall survival rates were $100 \%, 97.9 \%, 97.9 \%$ and $97.9 \%$, respectively. Age (>60 years old) was only prognostic factor with the p-value of 0.046 . For the toxicity profile, only $4.3 \%$ for GU could be found in our patients. No grade $3-4$ toxicity was observed. However, our study had some limitations. The numbers of patients was only 47 patients because at that time, using of IVBT as mono-therapy lacked supported study. Moreover, there were many treatment schedules of IVBT in our division at that period. Due to the effects of PORTEC-2 study, the using of IVBT increased and this affected to our policy by changing our schedule for adjuvant brachytherapy to $3 \times 7 \mathrm{~Gy}$. The results of $3 \times 7$ Gy schedule in our hospital will be further investigation to report the using of IVBT as monotherapy in endometrial cancer.

\section{Conclusion}

In conclusion, IVBT as monotherapy for endometrial cancer is feasible. There was only $4.3 \%$ of patients developed GU toxicity. Age was only prognostic factor in univariate analysis with the p-value of 0.046 .

Table 3. Late complication profiles of intravaginal brachytherapy.

\begin{tabular}{ccc}
\hline Parameters & Numbers (\%) \\
\hline Late skin & 0 \\
Late subcutaneous tissue & 0 \\
Late Gastrointestinal toxicity & 0 \\
Late Genitourinary toxicity & $2 / 47$ (4.3) \\
\hline
\end{tabular}




\section{Acknowledgements}

The author offers many thanks to Faculty of Medicine, Chiang Mai University and all staffs in the division to support this study.

\section{Funding}

This study was supported by Faculty of Medicine, Chiang Mai University.

\section{References}

[1] Tangjitgamol, S., Manusirivithaya, S., Srijaipracharoen, S., Khunnarong, J., Tanvanich, S., Katanyu, K., et al. (2010) Endometrial Cancer in Thai Women: Clinico-Pathological Presentation and Survival. Asian Pacific Journal of Cancer Prevention, 11, 1267-1272.

[2] Keys, H.M., Roberts, J.A., Brunetto, V.L., Zaino, R.J., Spirtos, N.M., Bloss, J.D., et al. (2004) A Phase III Trial of Surgery with or without Adjunctive External Pelvic Radiation Therapy in Intermediate Risk Endometrial Adenocarcinoma: A Gynecologic Oncology Group Study. Gynecologic Oncology, 92, 744-751. http://dx.doi.org/10.1016/j.ygyno.2003.11.048

[3] Creutzberg, C.L., van Putten, W.L., Koper, P.C., Lybeert, M.L., Jobsen, J.J., Wárlám-Rodenhuis, C.C., et al. (2000) Surgery and Postoperative Radio-Therapy versus Surgery Alone for Patients with Stage-1 Endometrial Carcinoma; Multicenter randomized Trial. Lancet, 355, 1404-1411. http://dx.doi.org/10.1016/S0140-6736(00)02139-5

[4] ASTEC/EN.5 Study Group, Blake, P., Swart, A.M., Orton, J., Kitchener, H., Whelan, T., Lukka, H., et al. (2009) Adjuvant External Beam Radiotherapy in the Treatment of Endometrial Cancer (MRC ASTEC and NCIC-CTG EN.5 Randomised Trials): Pooled Trial Results, Systematic Review, and Meta-Analysis. Lancet, 373, 137-146. http://dx.doi.org/10.1016/S0140-6736(08)61767-5

[5] Aalders, J., Abeler, V., Kolstad, P. and Onsrud, M. (1980) Postoperative External Irradiation and Prognostic Parameters in Stage I Endometrial Carcinoma: Clinical and Histopathologic Study of 540 Patients. Obstetrics \& Gynecology, 56, 419-427.

[6] Nout, R.A., Smit, V.T., Putter, H., Jürgenliemk-Schulz, I.M., Jobsen, J.J., Lutgens, L.C., et al. (2010) Vaginal Brachytherapy versus Pelvic External Beam Radiotherapy for Patients with Endometrial Cancer of High-Intermediate Risk (PORTEC-2): An Open-Label, Non-Inferiority, Randomised Trial. Lancet, 375, 816-823. http://dx.doi.org/10.1016/S0140-6736(09)62163-2

[7] Cox, J.D., Stetz, J. and Pajak, T.F. (1995) Toxicity Criteria of the Radiation Therapy Oncology Group (RTOG) and the European Organization for Research and Treatment of Cancer (EORTC). International Journal of Radiation Oncology*Biology* Physics, 31, 1341-1346. http://dx.doi.org/10.1016/0360-3016(95)00060-C

[8] Miller, A.B., Hoogstraten, B., Staquet, M. and Winkler, A. (1981) Reporting Results of Cancer Treatment. Cancer, 47, 207-214. http://dx.doi.org/10.1002/1097-0142(19810101)47:1<207::AID-CNCR2820470134>3.0.CO;2-6

[9] Kaplan, E.L. and Meier, P. (1958) Non-Parametric Estimation from Incomplete Observations. Journal of the American Statistical Association, 53, 457-481. http://dx.doi.org/10.1080/01621459.1958.10501452

[10] Mantel, N. (1966) Evaluation of Survival Data and Two New Rank Order Statistics Arising in Its Consideration. Cancer Chemotherapy Reports, 50, 163-170.

[11] Cox, D.R. (1972) Regression Models and Life Tables. Journal of the Royal Statistical Society: Series B, 34, 187-220.

[12] Hong, J.J., Yacoub, S.F., Blevins, J.C., Kuban, D.A. and El-Mahdi, A.M. (1997) High Dose Rate Vaginal Bracytherapyin Early Stage Endometrial Carcinoma: Preliminary Analysis. American Journal of Clinical Oncology, 20, 254-258. http://dx.doi.org/10.1097/00000421-199706000-00009

[13] Chadha, M., Nanavati, P.J., Liu, P., Fanning, J. and Jacobs, A. (1999) Patterns of Failure in Endometrial Carcinoma Stage IB Grade 3 and IC Patients Treated with Postoperative Vaginal Vault Brachytherapy. Gynecologic Oncology, 75, 103-107. http://dx.doi.org/10.1006/gyno.1999.5526

[14] McCloskey, S.A., Tchabo, N.E., Malhotra, H.K., Odunsi, K., Rodabaugh, K., Singhal, P., et al. (2010) Adjuvant Vaginal Brachytherapy Alone for High Risk Localized Endometrial Cancer as Defined by the Three Major Randomized Trials of Adjuvant Pelvic Radiation. Gynecologic Oncology, 116, 404-407.

http://dx.doi.org/10.1016/j.ygyno.2009.06.027

[15] Eldredge-Hindy, H.B., Eastwick, G., Anne, P.R., Rosenblum, N.G., Schilder, R.J., Chalian, R., et al. (2014) Adjuvant Vaginal Cuff Brachytherapy for High-Risk, Early Stage Endometrial Cancer. Journal of Contemporary Brachytherapy, 6, 262-270. http://dx.doi.org/10.5114/jcb.2014.45031 
Scientific Research Publishing (SCIRP) is one of the largest Open Access journal publishers. It is currently publishing more than 200 open access, online, peer-reviewed journals covering a wide range of academic disciplines. SCIRP serves the worldwide academic communities and contributes to the progress and application of science with its publication.

Other selected journals from SCIRP are listed as below. Submit your manuscript to us via either submit@scirp.org or Online Submission Portal.

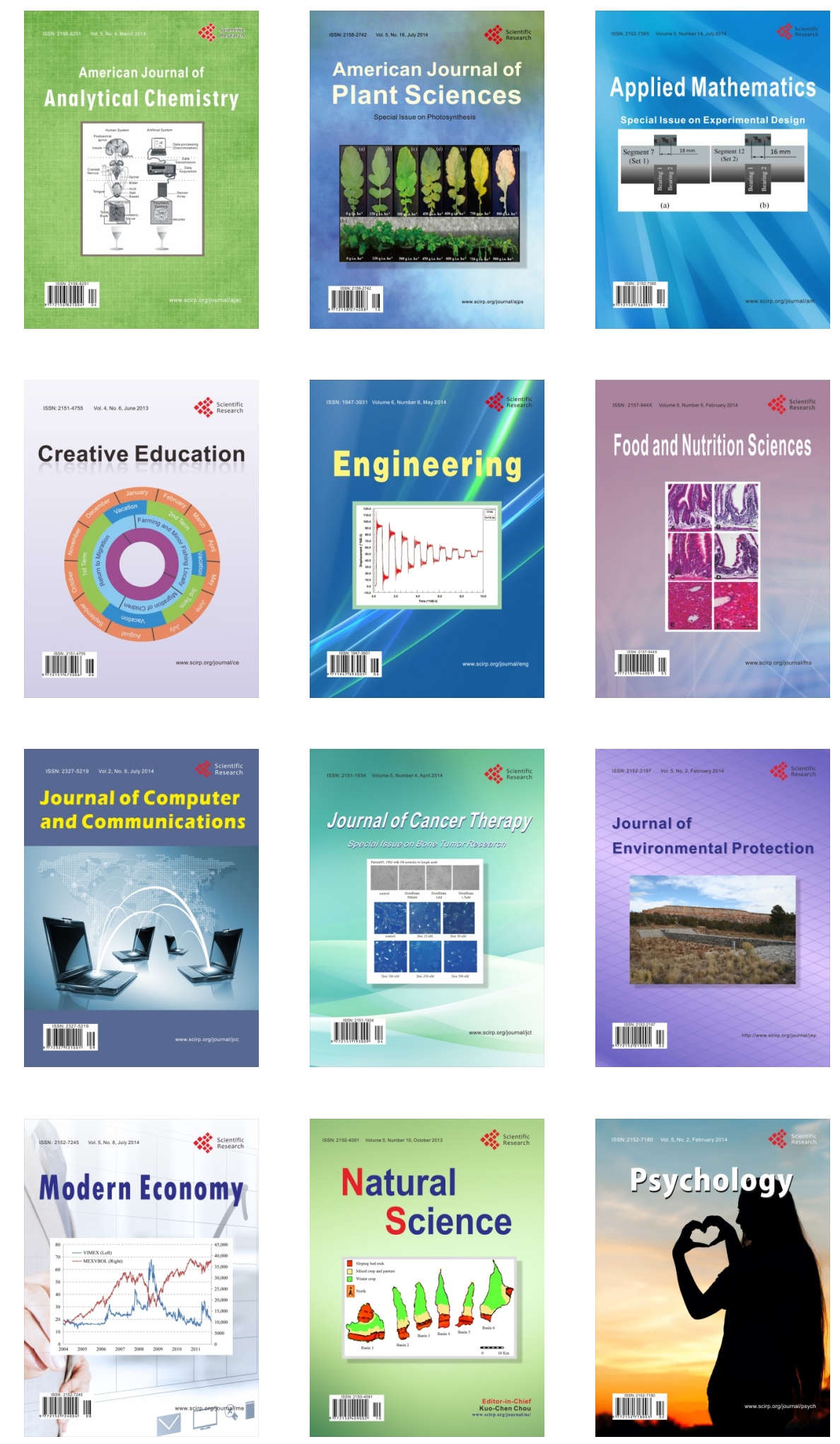